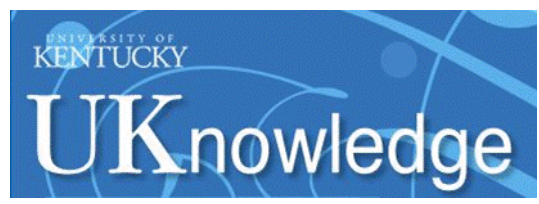

University of Kentucky

UKnowledge

$12-1-2016$

\title{
Prevalence of Triple-Negative Breast Cancer in India: Systematic Review and Meta-Analysis
}

\author{
Gurprataap S. Sandhu \\ University of Pittsburgh \\ Sebhat Erqou \\ University of Pittsburgh \\ Heidi Patterson \\ University of Pittsburgh
}

Aju Mathew

University of Kentucky, aju.mathew@uky.edu

Follow this and additional works at: https://uknowledge.uky.edu/markey_facpub

Part of the International and Area Studies Commons, and the Oncology Commons

Right click to open a feedback form in a new tab to let us know how this document benefits you.

\section{Repository Citation}

Sandhu, Gurprataap S.; Erqou, Sebhat; Patterson, Heidi; and Mathew, Aju, "Prevalence of Triple-Negative Breast Cancer in India: Systematic Review and Meta-Analysis" (2016). Markey Cancer Center Faculty Publications. 100.

https://uknowledge.uky.edu/markey_facpub/100

This Review is brought to you for free and open access by the Markey Cancer Center at UKnowledge. It has been accepted for inclusion in Markey Cancer Center Faculty Publications by an authorized administrator of UKnowledge. For more information, please contact UKnowledge@lsv.uky.edu. 
Prevalence of Triple-Negative Breast Cancer in India: Systematic Review and Meta-Analysis

Digital Object Identifier (DOI)

https://doi.org/10.1200/JG0.2016.005397

Notes/Citation Information

Published in Journal of Global Oncology, v. 2, issue 6, p. 412-421.

(C) 2016 by American Society of Clinical Oncology

Licensed under the Creative Commons Attribution 4.0 License. 


\section{Prevalence of Triple-Negative Breast Cancer in India: Systematic Review and Meta-Analysis}

Gurprataap S. Sandhu

Sebhat Erqou

Heidi Patterson

Aju Mathew

Gurprataap S. Sandhu, Sebhat Erqou, and Heidi Patterson, University of Pittsburgh Medical Center, Pittsburgh, PA; and Aju Mathew, Markey Cancer Center, University of Kentucky, Lexington, KY.

Authors' disclosures of potential conflicts of interest and contributions are found at the end of this article.

Corresponding author: Aju Mathew, MD, MPhil, University of Kentucky, 800 Rose St, CC447, Lexington, KY 40536; e-mail: aju.mathew@uky. edu.

Purpose There is considerable variation in prevalence rates of triple-negative breast cancer (TNBC) reported by various studies from India. We performed a systematic review and literature-based metaanalysis of these studies.

Methods We searched databases of Medline, Scopus, EMBASE, and Web of Science for studies that reported on the prevalence of TNBC in India that were published between January 1, 1999, and December 31,2015 . We extracted relevant information from each study by using a standardized form. We pooled study-specific estimates by using random-effects meta-analysis to provide summary estimates. We explored sources of heterogeneity by using subgroup analyses and metaregression.

Results Data were obtained from 17 studies that involved 7,237 patients with breast cancer. Overall combined prevalence of TNBC was $31 \%(95 \% \mathrm{CI}, 27 \%$ to $35 \%)$. There was substantial heterogeneity across the studies $\left(I^{2}\right.$ of $91 \%$ [95\% CI, $88 \%$ to $94 \%$ ]; $P<.001$ ) that was not explained by available study level characteristics, including study location, definition of human epidermal growth factor receptor 2 or estrogen receptor, mean age of participants, proportion of patients with premenopausal cancer, grade 3 disease, or tumor size $>5 \mathrm{~cm}$. Overall combined prevalence of hormone receptor-positive and human epidermal growth factor receptor 2-positive breast cancer was $48 \%$ (95\% Cl, $42 \%$ to $54 \%$ ) and $27 \%$ ( $95 \% \mathrm{CI}, 24 \%$ to $31 \%$ ), respectively. There was no evidence of publication bias.

Conclusion Prevalence of TNBC in India is considerably higher compared with that seen in Western populations. As many as as one in three women with breast cancer could have triple-negative disease. This finding has significant clinical relevance as it may contribute to poor outcomes in patients with breast cancer in India. Additional research is needed to understand the determinants of TNBC in India.

J Glob Oncol 2. (? 2016 by American Society of Clinical Oncology Licensed under the Creative Commons Attribution 4.0 License

\section{INTRODUCTION}

Breast cancer is a heterogeneous disease with different biologic subtypes that are recognized by gene expression profiling studies. Clinically, these subtypes are characterized on the basis of expression of estrogen receptor (ER), progesterone receptor (PR), and human epidermal growth factor receptor 2 (HER2). Triplenegative breast cancer (TNBC) is an aggressive subtype that is defined by lack of expression of ER and PR as well as absence of overexpressed or amplified HER2. ${ }^{1}$ TNBC accounts for approximately $12 \%$ to $17 \%$ of all invasive breast cancers in Western populations. ${ }^{1}$ TNBC occurs more frequently in younger women and is associated with higher histologic grade and more advanced disease. ${ }^{1,2}$ In North America, it is more frequently seen in women of African American ethnicity compared with other ethnic groups. ${ }^{2-5}$
Breast cancer is the most common cancer in India. ${ }^{6}$ For example, in 2012, it is estimated that approximately 145,000 new patients were diagnosed with breast cancer in India, and nearly 70,000 women died of the disease. ${ }^{7}$ Age-standardized 5-year breast cancer survival for Indian women diagnosed with breast cancer is $60 \%$ compared with $>80 \%$ in Western countries. ${ }^{8}$ Whereas breast cancer incidence seems to be increasing in the country, epidemiology of the disease is inadequately studied. ${ }^{9}$ Prevalence of TNBC in India is reported to be higher than that observed in Western populations; however, there is considerable variation in prevalence rates reported by studies from the region. It is important to obtain a reliable summary estimate of the prevalence of TNBC in India to address the growing burden of breast cancer in the country. Hence, we conducted a systematic review and 
literature-based meta-analysis of studies that reported on the prevalence of $\mathrm{TNBC}$ in India.

\section{METHODS}

\section{Search Strategy}

Studies that reported on the prevalence of TNBC in India that were published between January 1 , 1999, and December 31, 2015, were identified by using Medline, Scopus, EMBASE, and Web of Science databases (performed by G.S.S. and H.P.). Preferred Reporting Items for Systematic Reviews and Meta-Analyses guidelines were followed for article search and reporting. Computerbased search used combinations of the following medical subject headings and keywords: triple negative, breast cancer, and India.

\section{Study Eligibility}

We included studies in the meta-analysis if the following criteria were met: provided data on patients with breast cancer in India and described prevalence of triple-negative tumor subtype in the study population. We excluded studies that were comprised of patients with inflammatory breast cancer.

\section{Data Extraction}

Two investigators (G.S.S. and A.M.) independently screened all identified titles and abstracts from the search results. We scanned the reference lists of articles for additional articles. Full texts of screened articles were reviewed by G.S.S. and A.M. for inclusion criteria. Disagreements were resolved by consensus. Study selection process and flow diagram for identifying studies is detailed in Figure 1. We extracted relevant information from each study by using a standardized form, which was designed before implementation of the search strategy. The following information was extracted from each article by G.S.S. and A.M.: study design, region, publication year, study year, mean age, stage and grade distribution, receptor status, proportion of patients with ductal histology, proportion of patients with axillary lymph node involvement, proportion of patients who were premenopausal, and method of ER, PR, and HER2 assessment.

\section{Definitions of ER, PR, and HER2}

Level of ER is expressed as a product of the percentage of epithelial cells stained and intensity of staining through immunohistochemistry (IHC). Historically, the cutoff value of ER-positive disease was defined as nuclear staining of $\geqslant 10 \%$ of the epithelial component of the tumor; however, in 2010 ASCO lowered the IHC cutoff for determining
ER-positive status from the previous value of $10 \%$ to $1 \%$ of stained cells. ${ }^{10}$ This was based on the finding that even tumors weakly staining in $1 \%$ to $10 \%$ of cells demonstrated objective clinical benefitfrom treatment with tamoxifen. ${ }^{11}$ We extracted data on definitions of ER and PR positivity ( $\geqslant 1 \%$ or $\geqslant 10 \%$ or not reported).

With regard to HER2 testing, ASCO released an algorithm that defined positive, equivocal, and negative values for both HER2 protein expression and gene amplification. A positive HER2 result is $\mathrm{IHC}$ staining of $3+$ (uniform, intense membrane staining of $>30 \%$ of invasive tumor cells), a fluorescent in situ hybridization (FISH) result of more than six HER2 gene copies per nucleus, or a FISH ratio (HER2 gene signals to chromosome 17 signals) of more than 2.2. A negative result is an IHC staining of 0 or $1+$, a FISH result of fewer than 4.0 HER2 gene copies per nucleus, or a FISH ratio of less than 1.8. ${ }^{12}$ Equivocal results (IHC staining of $2+$ ) require additional testing for final classification. We extracted data on HER2 assessment method; studies that considered an IHC score of $2+$ and 3+ as positive for HER2, studies that considered an IHC score of $3+$ alone as positive for HER2, studies that used FISH test in patients who had 2+ score by $\mathrm{HC}$, and studies that did not disclose the method of checking HER2 status.

Only studies that reported on all three receptors (ER, PR, HER2) or those that reported on the proportion of TNBC were included in analyses. In some studies, more patients had data on ER or PR status than on HER2. Hence, in those studies, denominators were changed accordingly to provide an accurate assessment of the prevalence of hormone receptor (HR)-positive disease (defined as ER-positive and/or PR-positive), HER2-positive disease, or TNBC.

\section{Assessment of Study Quality}

We used STROBE statement guidelines to assess quality of selected studies. ${ }^{13}$ One point each was given if the study described the setting and study participants, reported descriptive data, provided detailed outcome data, and discussed limitations, for a maximum score of 5 and a minimum of zero. We performed subgroup analysis stratified by study quality score of $\geqslant 4$ versus $<4$.

\section{Statistical Analysis}

Standard errors for study-specific prevalence estimates were determined from the point estimate and appropriate denominators, assuming a binomial distribution. To obtain an overall summary estimate of prevalence across studies, we pooled 
Fig 1 -

Flow diagram for identifying studies for assessment of prevalence of triplenegative breast cancer in India.

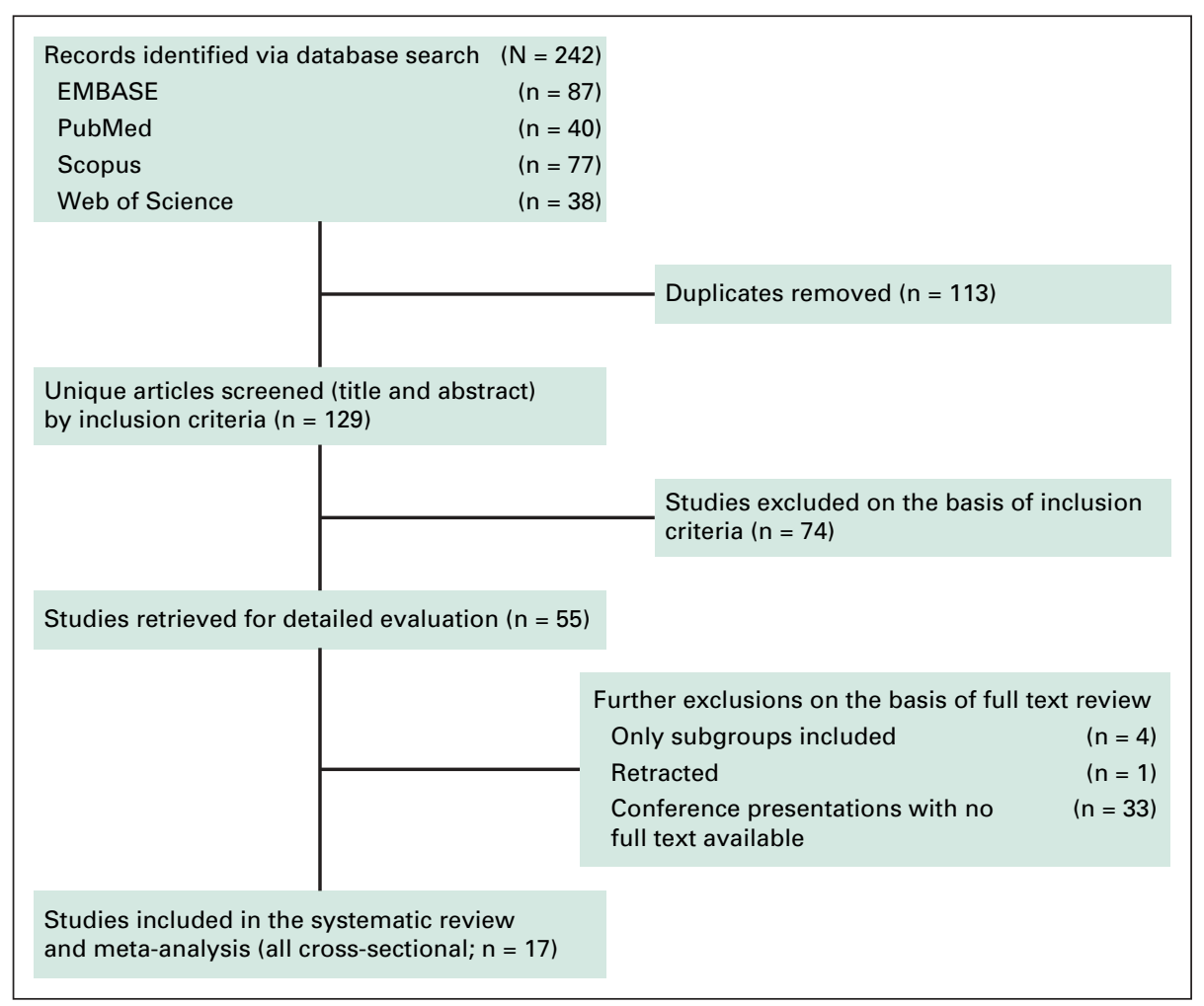

study-specific estimates by using a random-effects model meta-analysis. We also performed a fixedeffect model meta-analysis for comparison. Between-study heterogeneity was quantified by using $\mathrm{I}^{2}$ statistics. The $\mathrm{I}^{2}$ statistic estimates percentage of total variation across studies that are the result of true between-study differences rather than chance. We explored sources of heterogeneity by using subgroup analyses and metaregression. We assessed publication bias by using funnel plot symmetry and Egger regression intercept. All analyses were performed using STATA software (v13; STATA, College Station, TX; Computing Resource Center, Santa Monica, CA). Statistical tests were two-sided, and $P<.05$ was considered statistically significant.

\section{RESULTS}

\section{Study Characteristics}

Seventeen cross-sectional studies that involving 7,237 patients with breast cancer were included in this review (Table 1). ${ }^{14-30}$ Mean age of patients ranged from 43 to 55 years across studies, with a weighted average of age 50 years. Proportion of patients who were premenopausal ranged from $27 \%$ to $67 \%$ across studies, with a weighted average of $41 \%$. Proportion of patients with grade 3 disease ranged from $16 \%$ to $75 \%$, with a weighted average of $57 \%$; corresponding figures for patients with tumor size $>5 \mathrm{~cm}$ were $3 \%$ to $65 \%$ (weighted average,
24\%) and those for patients with positive axillary lymph nodes were 39\% to 90\% (weighted average, $57 \%$ ). Prevalence estimates of HR-positive disease, HER2-positive disease, and TNBC in each of the included studies are reported in Table 1.

\section{Prevalence of TNBC}

Overall combined prevalence of TNBC in the random-effects model meta-analysis was 31\% (95\% Cl, 27\% to 35\%). Pooled estimate from the fixed-effect model meta-analyses was comparable (Fig 2). There was substantial heterogeneity across the studies ( ${ }^{2}$ of $91.2 \%$ [95\% Cl, 88\% to 94\%]; $P<.001)$. Sources of heterogeneity were explored by several study-level characteristics, as described in Prevalence of TNBC by Study-level Characteristics. Overall combined prevalence of HR-positive breast cancer was $48 \%$ (95\% Cl, $42 \%$ to $54 \%$; Fig 3), and overall combined prevalence of HER2-positive breast cancer was 27\% (95\% Cl, 24\% to 31\%; Fig 4).

\section{Prevalence of TNBC by Study-Level Characteristics}

In an attempt to explain heterogeneity, we performed subgroup analysis and metaregression by using several study-level characteristics (Fig 5). Meta-analysis included four studies each from the north, east, and west of the country and five studies 


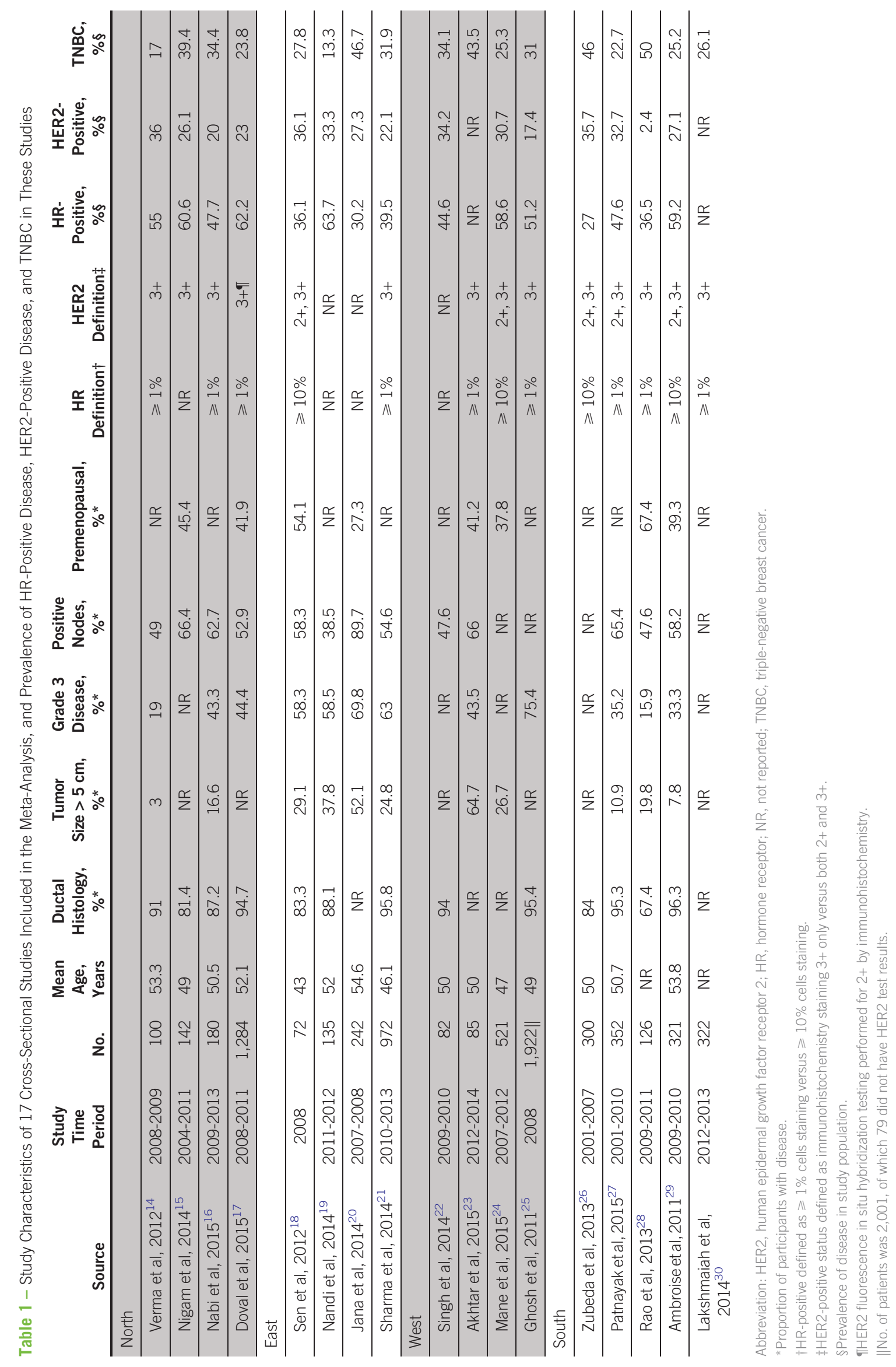


Fig 2 -

Prevalence of triplenegative breast cancer across 17 studies in India. E, east; N, north; S, south; $\mathrm{W}$, west.
Fig 3 -

Prevalence of hormone receptor-positive breast cancer across 17 studies in India. E, east; N, north; S, south; W, west.

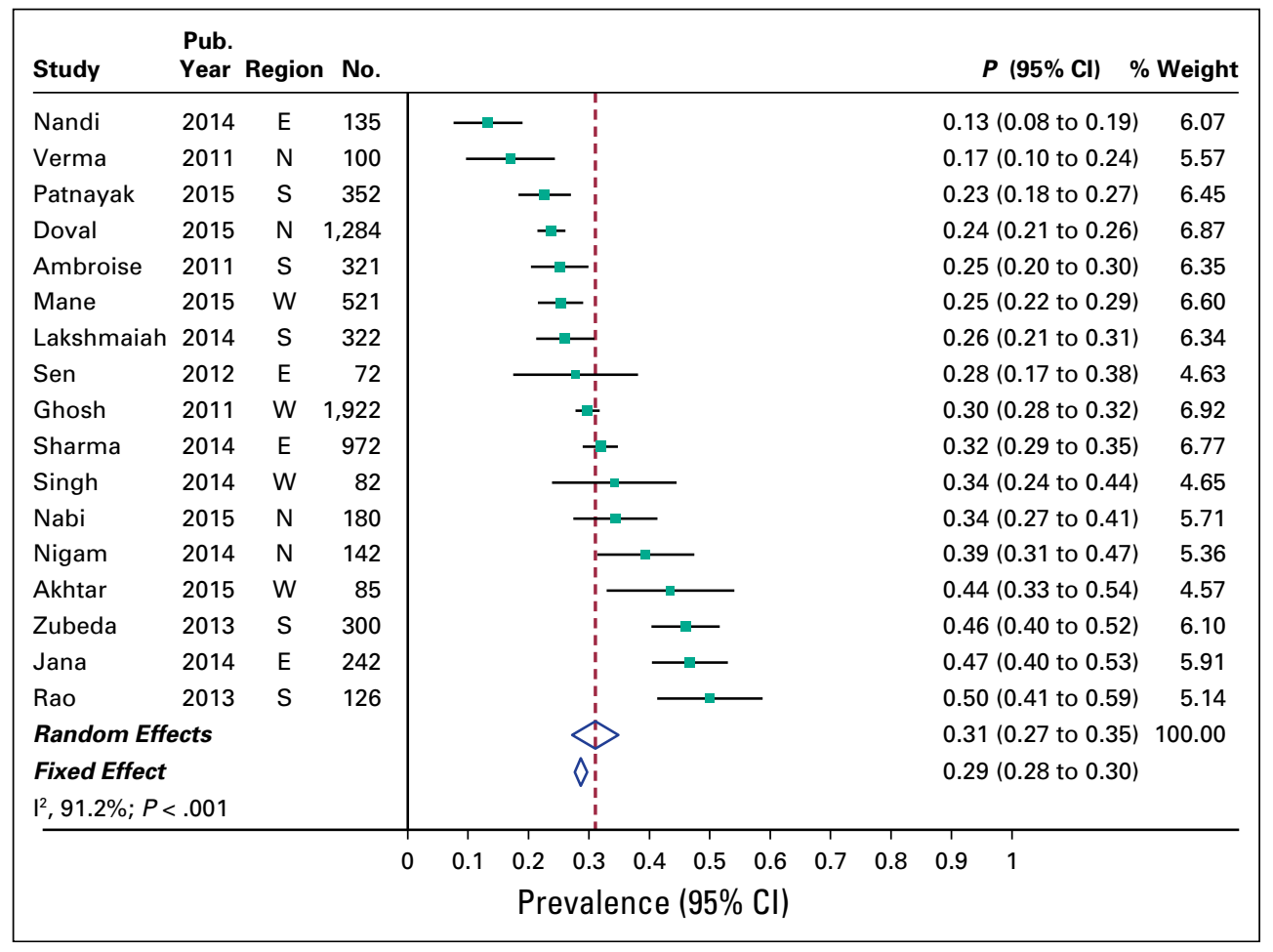

from the south. We found no significant difference in prevalence of TNBC by region. Overall prevalence of TNBC in the north, south, east, and west of the country was $28 \%, 34 \%, 30 \%$, and $31 \%$, respectively. Heterogeneity in overall prevalence of TNBC was not explained by other available study-level characteristics, such as mean age of participants or proportion of patients who were premenopausal, had grade 3 disease, or had tumor size $>5 \mathrm{~cm}$.

\section{Definition of ER, PR, and HER2}

Definition of HR and HER2 was variable across studies (Table 1). Nine studies defined HR-

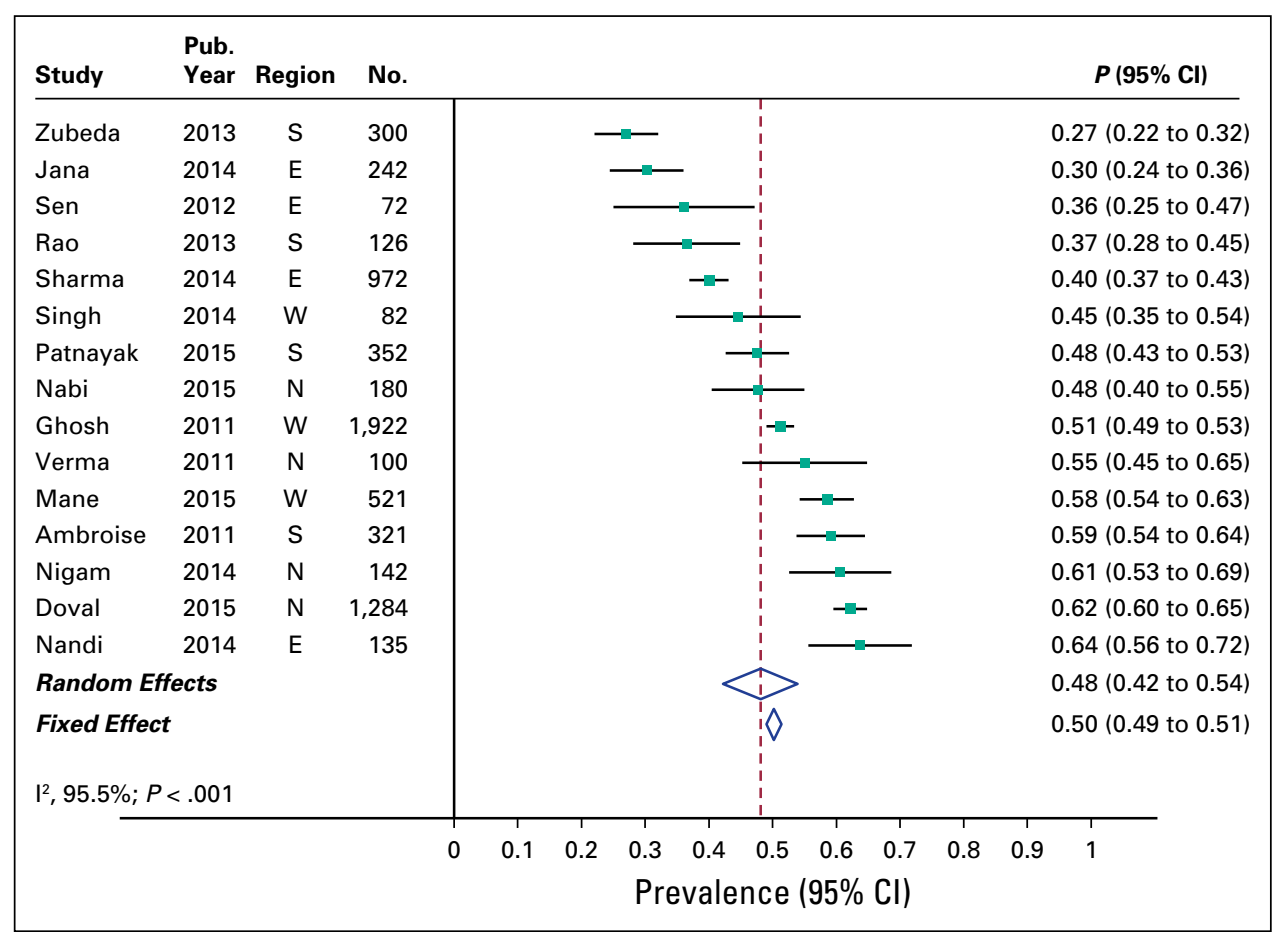


Fig 4 -

Prevalence of human epidermal growth factor receptor 2-positive breast cancer across 17 studies in India. E, east; N, north; S, south; W, west.

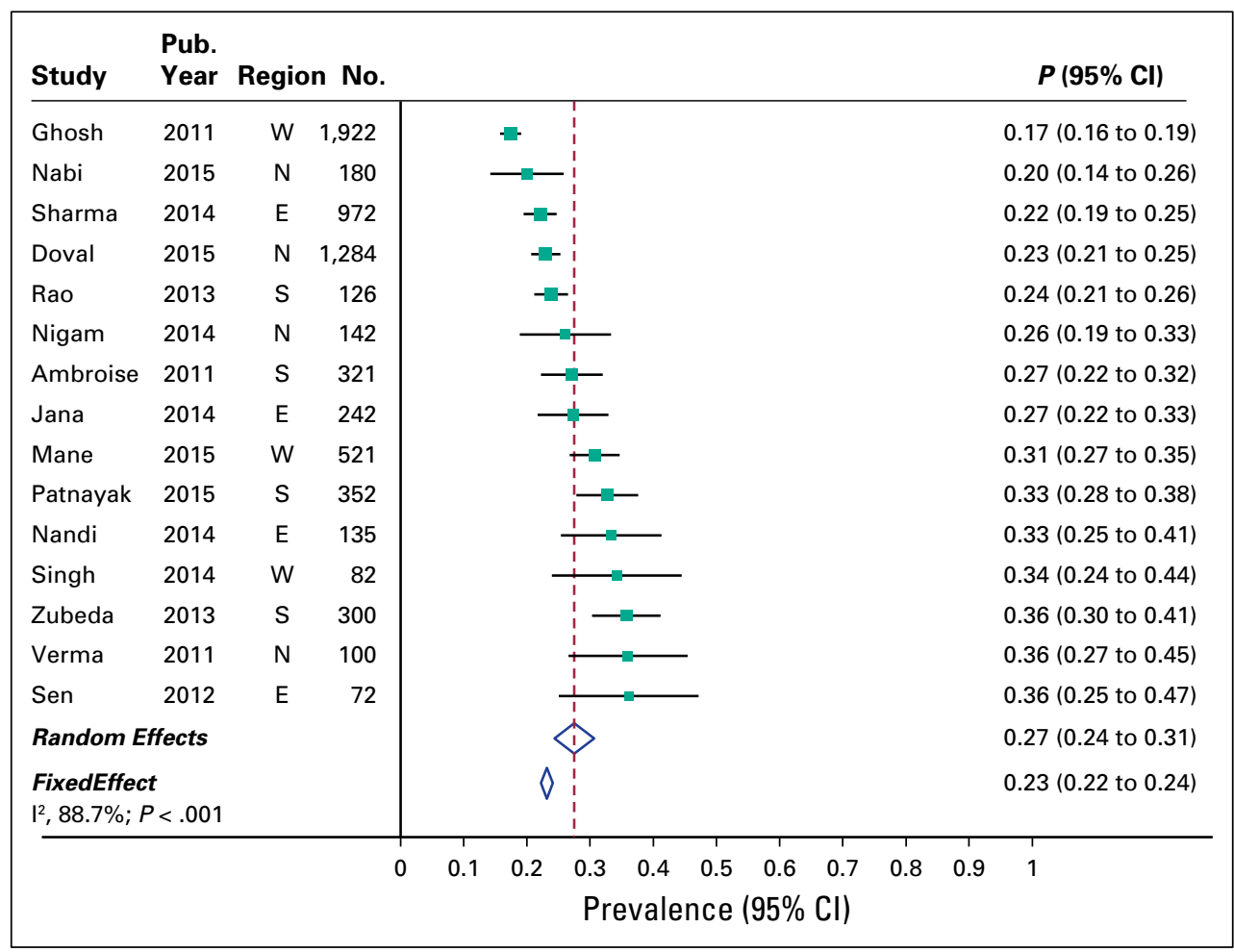

positive as ER and $P R \geqslant 1 \%$ and four studies defined HR-positive as ER and PR $\geqslant 10 \%$, whereas four studies did not describe the definition of HR. Nine studies defined HER2-positive status as only $3+$ by IHC (Doval et al ${ }^{17}$ used FISH test to classify equivocal cases and is therefore included in the 3+ group) and five studies included $2+$ as positive, whereas three studies did not describe the definition of HER2. We did not find significant difference in pooled estimates subgrouped by different definitions for HR and HER2 (Fig 5).

\section{Study Quality}

One study scored the maximum score of 5,11 studies scored 4 , four studies scored 3 , and one study scored 2 on assessment of study quality. Lower quality studies (study quality score $<4$ ) had a tendency to report higher prevalence of TNBC compared with higher quality studies (score $\geqslant 4$ ); $38 \%$ [95\% Cl, 27\% to 48\%] versus $29 \%$ [95\% Cl, 25\% to 33\%], respectively, although confidence intervals overlap $(P=.12$; Fig 5).

\section{Assessment of Publication Bias}

There was no evidence of publication bias by visual inspection of funnel plot or Egger test for bias ( $P=$.24; Appendix Fig A1). Furthermore, pooled estimate from larger-sized studies $(N \geqslant 250)$, which are less likely to be subjected to publication bias, with that from smaller-sized studies $(\mathrm{N}<250)$ were comparable (29\% [95\% Cl, 25\% to 32\%] versus $34 \%$ [95\% Cl, 25\% to 43\%]; $P=.34$; Fig 4).

\section{Sensitivity Analysis}

Rao et al ${ }^{28}$ described a low prevalence of HER2positive disease $(2.4 \% ; n=126)$. Prevalence of TNBC in this study was $50 \%$. Such a low rate of HER2-positive disease has not been reported from India or elsewhere; however, a sensitivity analysis performed after omitting data from the study yielded results that were similar to the main analyses.

\section{DISCUSSION}

To our knowledge, this systematic review and meta-analysis of 17 cross-sectional studies that involved 7,237 patients with breast cancer is the most comprehensive analysis on prevalence of TNBC in India. Prevalence of TNBC in India ranged from $27 \%$ to $35 \%$ across studies, with a summary estimate of $31 \%$. This is comparable to the prevalence seen in African American women and is more than twice the rate seen in white women. ${ }^{5,31-34}$ Our study also found a higher prevalence of premenopausal breast cancer, grade 3 disease, and larger tumor size, all of which are associated with triple-negative disease. ${ }^{1-3} \mathrm{Be}$ cause TNBC is known to be more aggressive than other breast cancer subtypes, higher prevalence 
Fig 5 -

Prevalence of triple negative breast cancer by various study characteristics. ER, estrogen receptor; HER2, human epidermal growth factor receptor 2 .

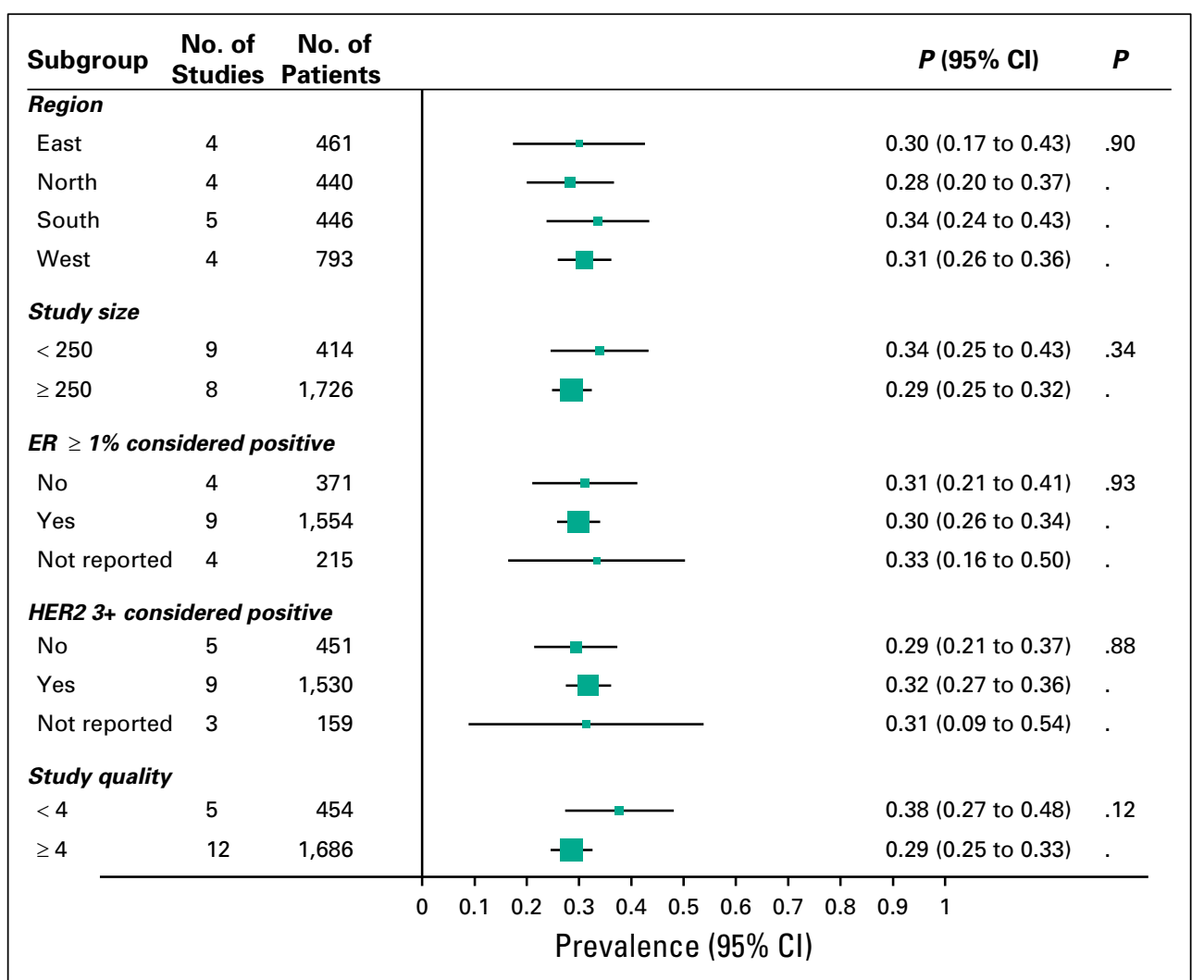

of TNBC could be a contributing factor to the high fatality rate of patients with breast cancer in India.

We explored sources of heterogeneity through subgroup analyses by using study-level characteristics that are known to be associated with a higher prevalence of TNBC, but results were not statistically significant. We also analyzed data on the basis of study location and found similar prevalence rates for TNBC in all four regions in India. We also found that differences in definitions of $\mathrm{HR}$ and HER2 positivity did not influence study results.

One limitation of the present meta-analysis is the presence of substantial heterogeneity across the contributing studies that is unexplained by available study-level characteristics; however, similarity of findings from pooled estimates from random-effects and fixed-effect models is reassuring. Furthermore, pooled estimates from the smalland larger-sized studies were comparable. India is a vast country with significant variations in linguistic, geographic, dietary, cultural, and health careassociated practices; therefore, one can postulate the presence of considerable inherent heterogeneity at state level that was not captured by regional compartmentalization. Second, we did not incorporate quality scores into meta-analysis weights. An analysis by study quality suggested a higher prevalence of TNBC in lower-quality studies; however, difference in prevalence rates by study quality was not statistically significant. Third, the contributing study results are subjected to local laboratory standards and practices. Fourth, primary data are obtained from studies that were conducted at various tertiary-level hospitals, which could result in an inherent selection bias as a result of the possibility that patients who have more advanced disease could be selectively referred to such institutions. However, most cancer care in India occurs in tertiary-level institutions, and, therefore, we do not think the actual prevalence rate will be significantly different from that identified in our meta-analysis. Finally, several potential risk factors, such as obesity, parity, and socioeconomic factors, have not been described in the studies that are included in this meta-analysis.

Multiple factors may account for higher prevalence of TNBC reported by studies conducted among Indian patients with breast cancer. The early age of onset of breast cancer; lifestyle factors, such as diet and obesity; reproductive factors, such as multiparity; socioeconomic status; and screening behaviors may be hypothesized as probable etiology. ${ }^{2,3}$ Another important factor could be a potential genetic susceptibility of Indians to TNBC. More focused research into these factors will help clarify underlying determinants of TNBC in India. A first step in this direction would 
be to institute a prospectively managed, population-based database of patients with breast cancer, along with reliable histopathologic testing.

In conclusion, this systematic review and metaanalysis demonstrated a high prevalence rate of TNBC in India. Further research to identify the determinants of such a high prevalence of TNBC is warranted as they may have implications for addressing the burden of breast cancer mortality in India.

\section{DOI: 10.1200/JGO.2016.005397}

Published online on ascopubs.org/journal/jgo on June 29, 2016.

\section{AUTHOR CONTRIBUTIONS}

Conception and design: Gurprataap S. Sandhu, Aju Mathew Collection and assembly of data: Gurprataap S. Sandhu, Heidi Patterson, Aju Mathew

Data analysis and interpretation: Gurprataap S. Sandhu, Sebhat Erqou, Aju Mathew

Manuscript writing: All authors

Final approval of manuscript: All authors

Accountable for all aspects of the work: All authors

\section{AUTHORS' DISCLOSURES OF}

POTENTIAL CONFLICTS OF INTEREST

The following represents disclosure information provided by authors of this manuscript. All relationships are considered compensated. Relationships are self-held unless noted.
I = Immediate Family Member, Inst = My Institution. Relationships may not relate to the subject matter of this manuscript. For more information about ASCO's conflict of interest policy, please refer to www.asco.org/rwc or ascopubs.org/jco/ site/ifc.

Gurprataap S. Sandhu

No relationship to disclose

Sebhat Erqou

No relationship to disclose

Heidi Patterson

No relationship to disclose

Aju Mathew

No relationship to disclose

\section{REFERENCES}

1. Foulkes WD, Smith IE, Reis-Filho JS: Triple-negative breast cancer. N Engl J Med 363:1938-1948, 2010

2. Boyle P: Triple-negative breast cancer: Epidemiological considerations and recommendations. Ann Oncol 23 (suppl 6):vi7-vi12, 2012

3. Brewster AM, Chavez-MacGregor M, Brown P: Epidemiology, biology, and treatment of triple-negative breast cancer in women of African ancestry. Lancet Oncol 15:e625-e634, 2014

4. Bauer KR, Brown M, Cress RD, et al: Descriptive analysis of estrogen receptor (ER)-negative, progesterone receptor (PR)-negative, and HER2-negative invasive breast cancer, the so-called triple-negative phenotype: A populationbased study from the California Cancer Registry. Cancer 109:1721-1728, 2007

5. Carey LA, Perou CM, Livasy CA, et al: Race, breast cancer subtypes, and survival in the Carolina Breast Cancer Study. JAMA 295:2492-2502, 2006

6. Fitzmaurice C, Dicker D, Pain A, et al: The global burden of cancer 2013. JAMA Oncol 1:505-527, 2015 [Erratum: JAMA Oncol 1:690, 2015]

7. International Agency for Research on Cancer: GLOBOCAN 2012: Estimated cancer incidence, mortality and prevalence worldwide in 2012. http://globocan.iarc.fr/Pages/fact_sheets_cancer.aspx

8. Allemani C, Weir HK, Carreira H, et al: Global surveillance of cancer survival 1995-2009: Analysis of individual data for 25,676,887 patients from 279 population-based registries in 67 countries (CONCORD-2). Lancet 385:977-1010, 2015

9. Khokhar A: Breast cancer in India: Where do we stand and where do we go? Asian Pac J Cancer Prev 13:4861-4866, 2012

10. Gown AM: Current issues in ER and HER2 testing by IHC in breast cancer. Mod Pathol 21:S8-S15, 2008 (suppl 2)

11. Davies C, Godwin J, Gray R, et al: Relevance of breast cancer hormone receptors and other factors to the efficacy of adjuvant tamoxifen: Patient-level meta-analysis of randomised trials. Lancet 378:771-784, 2011

12. Wolff AC, Hammond MEH, Schwartz JN, et al: American Society of Clinical Oncology/College of American Pathologists guideline recommendations for human epidermal growth factor receptor 2 testing in breast cancer. Arch Pathol Lab Med 131:18-43, 2007

13. Vandenbroucke JP, von Elm E, Altman DG, etal: Strengthening the Reporting of Observational Studies in Epidemiology (STROBE): Explanation and elaboration. Ann Intern Med 147:W163-W194, 2007

14. Verma S, Bal A, Joshi K, et al: Immunohistochemical characterization of molecular subtypes of invasive breast cancer: A study from North India. APMIS 120:1008-1019, 2012

15. Nigam JS, Yadav P, Sood N: A retrospective study of clinico-pathological spectrum of carcinoma breast in a West Delhi, India. South Asian J Cancer 3:179-181, 2014 
16. Nabi MG, Ahangar A, Wahid MA, et al: Clinicopathological comparison of triple negative breast cancers with non-triple negative breast cancers in a hospital in North India. Niger J Clin Pract 18:381-386, 2015

17. Doval DC, Sharma A, Sinha R, et al: Immunohistochemical profile of breast cancer patients at a tertiary care hospital in New Delhi, India. Asian Pac J Cancer Prev 16:4959-4964, 2015

18. Sen S, Gayen R, Das S, et al: A clinical and pathological study of triple negative breast carcinoma: Experience of a tertiary care centre in eastern India. J Indian Med Assoc 110:686-689, 705, 2012

19. Nandi M, Mahata A, Mallick I, et al: Hypofractionated radiotherapy for breast cancers-Preliminary results from a tertiary care center in eastern India. Asian Pac J Cancer Prev 15:2505-2510, 2014

20. Jana D, Sarkar DK, Ganguly S, et al: Can molecular subtyping replace axillary nodal status as prognostic marker in breast cancer? Indian J Surg Oncol 5:282-289, 2014

21. Sharma M, Sharma JD, Sarma A, et al: Triple negative breast cancer in people of North East India: Critical insights gained at a regional cancer centre. Asian Pac J Cancer Prev 15:4507-4511, 2014

22. Singh R, Gupta S, Pawar SB, et al: Evaluation of ER, PR and HER-2 receptor expression in breast cancer patients presenting to a semi urban cancer centre in Western India. J Cancer Res Ther 10:26-28, 2014

23. Akhtar M, Dasgupta S, Rangwala M: Triple negative breast cancer: An Indian perspective. Breast Cancer (Dove Med Press) 7:239-243, 2015

24. Mane A, Khatib KI, Deshmukh SP, et al: A comparison of clinical features, pathology and outcomes in various subtypes of breast cancer in Indian women. J Clin Diagn Res 9:PC01-PC04, 2015

25. Ghosh J, Gupta S, Desai S, et al: Estrogen, progesterone and HER2 receptor expression in breast tumors of patients, and their usage of HER2-targeted therapy, in a tertiary care centre in India. Indian J Cancer 48:391-396, 2011

26. Zubeda S, Kaipa PR, Shaik NA, et al: Her-2/neu status: A neglected marker of prognostication and management of breast cancer patients in India. Asian Pac J Cancer Prev 14:2231-2235, 2013

27. Patnayak R, Jena A, Rukmangadha N, et al: Hormone receptor status (estrogen receptor, progesterone receptor), human epidermal growth factor-2 and p53 in South Indian breast cancer patients: A tertiary care center experience. Indian J Med Paediatr Oncol 36:117-122, 2015

28. Rao C, Shetty J, Kishan Prasad HL: Morphological profile and receptor status in breast carcinoma: An institutional study. J Cancer Res Ther 9:44-49, 2013

29. Ambroise M, Ghosh M, Mallikarjuna VS, et al: Immunohistochemical profile of breast cancer patients at a tertiary care hospital in South India. Asian Pac J Cancer Prev 12:625-629, 2011

30. Lakshmaiah KC, Das U, Suresh TM, et al: A study of triple negative breast cancer at a tertiary cancer care center in southern India. Ann Med Health Sci Res 4:933-937, 2014

31. Trivers KF, Lund MJ, Porter PL, et al: The epidemiology of triple-negative breast cancer, including race. Cancer Causes Control 20:1071-1082, 2009

32. Amirikia KC, Mills $P$, Bush J, et al: Higher population-based incidence rates of triple-negative breast cancer among young African-American women: Implications for breast cancer screening recommendations. Cancer 117:27472753, 2011

33. Stead LA, Lash TL, Sobieraj JE, et al: Triple-negative breast cancers are increased in black women regardless of age or body mass index. Breast Cancer Res 11:R18, 2009

34. Stark A, Kleer CG, Martin I, et al: African ancestry and higher prevalence of triple-negative breast cancer: Findings from an international study. Cancer 116:4926-4932, 2010 
Fig A1 -

Funnel plot with pseudo 95\% Cls. TNBC, triplenegative breast cancer.

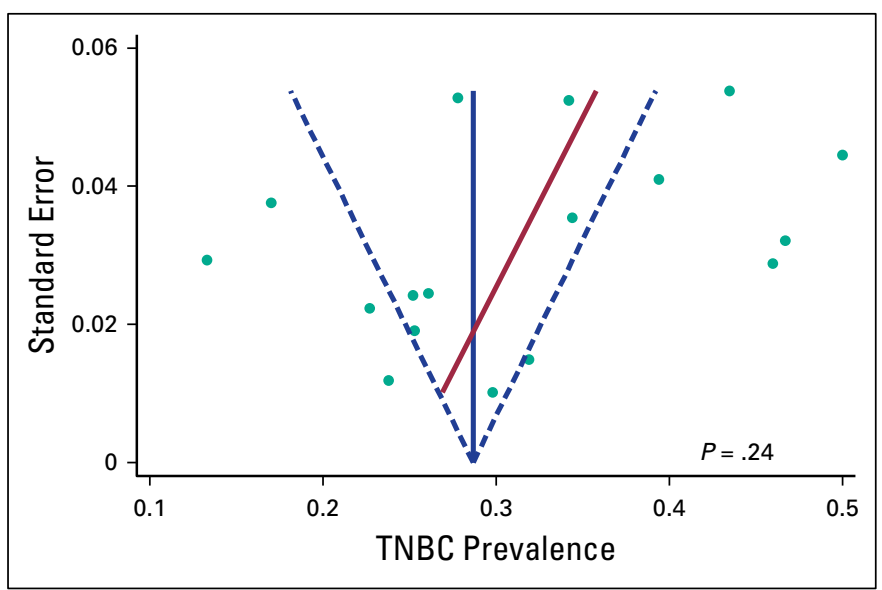

